\title{
Investigating the Relationship Between Personality Dimensions and Choice of Type of Delivery in Patients Referred to Hazrat Abolfazl Hospital in Minab County
}

\author{
Zahra Mohammad Alipour $^{\circledR}{ }^{\circledR}$, Misa Naghdipour Mirsadeghi², Hadi Marzban', Seyed Hamzeh Seddigh ${ }^{(\circledR}$, Ali $^{1}$ \\ Massoudifar $^{3}$ \\ ${ }^{1}$ Student Research Committee, Faculty of Medicine, Hormozgan University of Medical Sciences, Bandar Abbas, Iran. \\ ${ }^{2}$ Department of Obstetrics and Gynecology, Faculty of Medicine, Hormozgan University of Medical Sciences, Bandar \\ Abbas, Iran. \\ ${ }^{3}$ Department of Psychiatry, Faculty of Medicine, Hormozgan University of Medical Sciences, Bandar Abbas, Iran.
}

\begin{abstract}
Background: Human choices and decisions are highly affected by some psychological and personality traits. These psychological characteristics may also be related to the choice of delivery type. Therefore, the present study aimed to investigate the relationship between personality dimensions and choice of delivery type (cesarean section or normal) in pregnant women referred to Abolfazl hospital in Minab county, Iran, in 2018.

Materials and Methods: This is a cross-sectional descriptive study. The statistical population consisted of all pregnant women referred to Abolfazl medical center in 2008 in Minab, 118 of whom were selected by convenience sampling. The NEO Five-Factor Inventory (NEO-FFI) was used to collect the data.

Results: There was a significant difference in the choice of delivery type (normal and cesarean section) in pregnant women with extraversion and conscientiousness personality traits ( $P$ value $<0.05$ ). The mean extraversion and conscientiousness scores were higher in patients who tended to have a vaginal delivery than in those who had chosen cesarean section.

Conclusion: The results of the present study show that personality traits can determine the type of delivery by creating specific moods; therefore, considering the high level of complications of cesarean section (CS) and high CS rate in Iran, we can change people's attitudes and play a role in reducing the number of CSs through long-term training.

Keywords: Type of delivery, Personality traits, Cesarean section, Neo FFI , Normal delivery
\end{abstract}

*Correspondence to Ali Massoudifar, Department of Psychiatry, Faculty of Medicine,

Hormozgan University of Medical Sciences, Bandar Abbas, Iran. Tel: +989151003882 Email: ali.massoudifar@ gmail.com

Received: October 10, 2019, Accepted: November 24, 2019, ePublished: December 15, 2019

\section{Introduction}

Delivery is a physiological process, the grounds and condition of which have been made available in human beings like other mammals by God so they do the delivery normally without medical intervention. Eighty-five percent of deliveries do not require medical intervention, and this physiological and natural process occurs successfully only by providing training and care for mothers and monitoring them during the process (1-3). Choosing the right delivery method worries each couple when they are expecting a baby that can affect the health of the mother and baby. Over the last few decades, there was an increasing growth in cesarean section (CS) rate in most countries, especially Iran, due to non-obstetric factors, among which the fear of labor pain is the most important $(1,3,4,5)$. Since labor has a painful nature, mothers' reaction to labor pain varies depending on factors such as culture, fear and worry, previous delivery experience, readiness for delivery, and spousal support (5-7). Fear of labor pain has been suggested as an important factor leading to a decrease in normal vaginal delivery and increase in CS rate, and maternal personality traits can play an essential and significant role in the occurrence of such fear (1, 8-10). Since personality is a complex psychological construct, it exhibits a variety of human behaviors in different situations. McCrae et al referred to neuroticism, extraversion, openness to experience, adaptability, and 
responsibility as five dimensions of personality model (11). Since the personality traits of pregnant women are important determinants of the type of delivery, we can provide a framework for pregnant women's behavior regarding the choice of delivery type and the reduction of CS rate by identifying factors affecting these traits (1, 12). The aim of the present study was to investigate the differences in personality traits and anxiety of pregnant women based on choice of delivery type. Accordingly, we identify the personality traits of pregnant women and the effective factors and thus can play a role in reducing CS rates by providing long-term training and changing their attitude.

\section{Materials and Methods}

This is a descriptive cross-sectional study that was performed on 118 patients referred to gynecology ward of Abolfazl hospital in Minab county for delivery during June, July, and August of 2018. Data collection instrument was NEO Five-Factor Inventory (NEO-FFI) (11). Data collection was carried out using field and library methods. NEO-FFI (11) was used after obtaining the approval of the Ethics Committee and ensuring all ethical considerations, including non-compulsory evaluation and informed consent to assess personality traits (11). The above-mentioned inventory measures demographic variables such as age, level of education, place of residence (urban or rural), previous experience of CS or normal delivery, choice of delivery type, choice of the infant's gender, choice of having one's husband in the labor, and previous pregnancy planning .(Table 1). The answer sheet of the above-mentioned inventory was designed based on a Likert scale (Strongly disagree, Disagree, Indifferent, Agree, and Strongly agree). A short form of NEO-FFI was used; therefore, some items of it are scored differently as follows: Strongly disagree (Score 4), Disagree (Score 3), Indifferent (Score 2), Agree (Score 1), and Completely agree (Score 0). However, some of the other items in this short form are scored reversely. Subjects were then divided into five personality types (neuroticism, Agreeableness, openness to experience, extraversion, and conscientiousness) based on the score obtained (Table 2). Finally, data analysis was carried out using descriptive statistics (mean and standard deviation), and MANOVA inferential statistics by SPSS version 22.0.

\section{Results}

Neo-FFI was used to collect data of 118 patients who participated in the study. The mean age of participants was $28.83 \pm 6.01$ years and their age ranged from 15 to 43 years.

The participants were divided into 5 personality groups (extroversion, openness to experience, agreeableness, conscientiousness, and neuroticism) ( Table 2).

Participants with extraversion and conscientiousness personality traits had a higher tendency to have a vaginal delivery than CS and there was a significant relationship between personality dimensions and type of delivery (Table 2).

The present study showed a significant difference in the choice of the type of infant's gender between neuroticism $(P$ value $=0.003)$ and openness to experience $(P$ value $=0.39$ ), but there was no significant difference between other dimensions. Pregnant women with higher mean neuroticism scores tended to have a female infant and women who had higher scores on openness to experience tended to have a male one (Table 2).

There was a statistically significant difference between openness to experience, agreeableness, and extraversion in terms of the choice of delivery type based on the patient's

Table 1. Frequency Distribution Table for Demographic Variables

\begin{tabular}{|c|c|c|c|}
\hline Variables & & Number & Percent \\
\hline \multirow{4}{*}{ Level of education } & Elementary & 10 & 8.5 \\
\hline & Middle school & 17 & 14.4 \\
\hline & Diploma & 41 & 34.7 \\
\hline & Associate degree and above & 50 & 42.4 \\
\hline \multirow{2}{*}{ Place of residence } & City & 103 & 87.3 \\
\hline & Village & 15 & 12.7 \\
\hline \multirow{2}{*}{ Choice of delivery type } & Normal & 84 & 71.2 \\
\hline & Cesarean section & 34 & 28.8 \\
\hline \multirow{2}{*}{ Choice infant's gender } & Male & 50 & 42.4 \\
\hline & Female & 68 & 57.6 \\
\hline \multirow{2}{*}{ Choice of having one's husband in the labor } & Yes & 101 & 85.6 \\
\hline & No & 17 & 14.4 \\
\hline \multirow{3}{*}{ Previous delivery } & Normal & 40 & 33.9 \\
\hline & Cesarean section & 29 & 24.6 \\
\hline & None & 49 & 41.5 \\
\hline \multirow{2}{*}{ Previous pregnancy planning } & Yes & 73 & 61.9 \\
\hline & No & 45 & 38.1 \\
\hline
\end{tabular}


Table 2. Comparison of the Mean and Range of Changes in Personality Dimensions in Terms of Demographic Variables

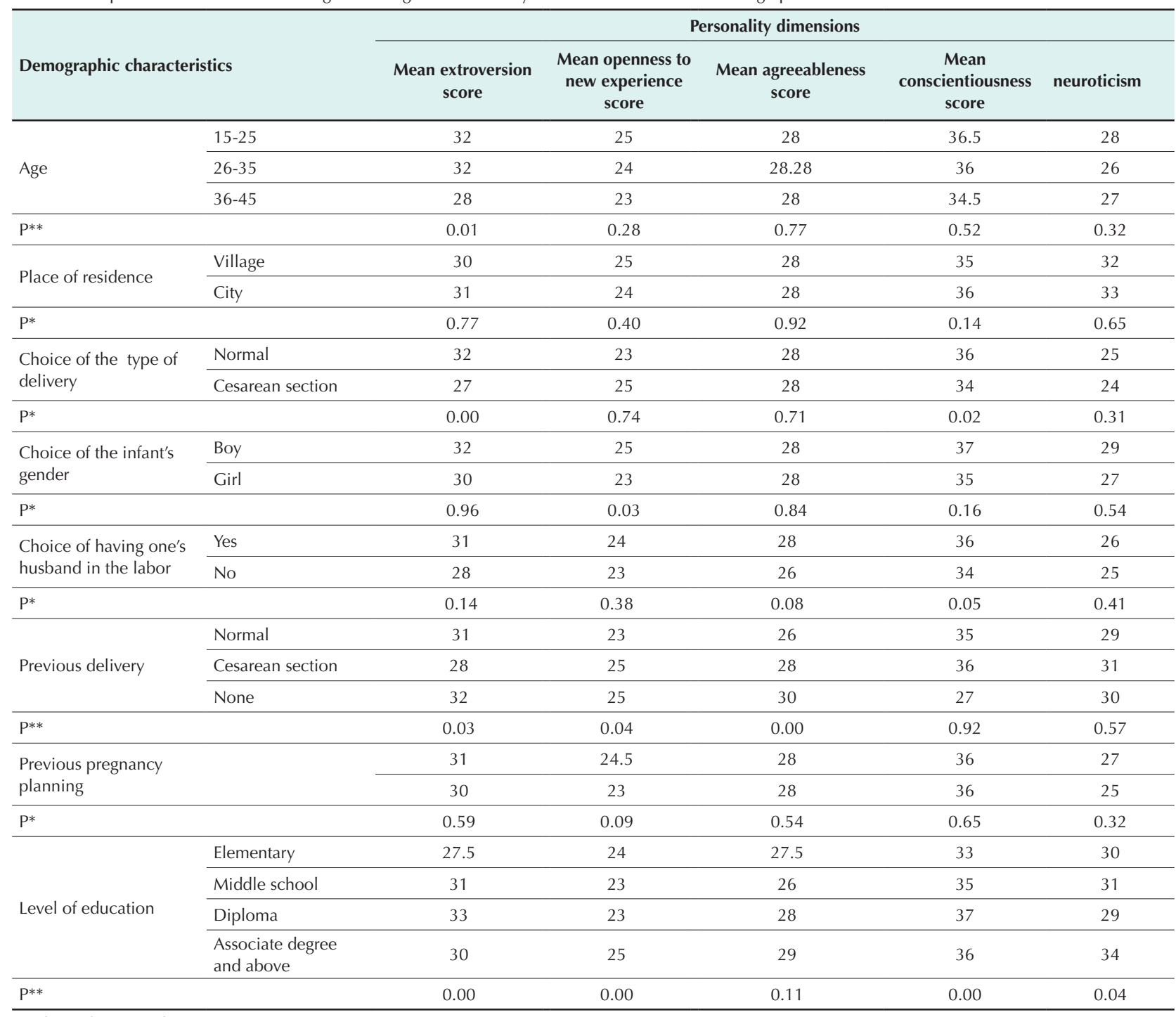

* Independent Sample T Test

**One Way ANOVA

previous history of delivery, but there was no significant difference in other dimensions. Pregnant women who did not have previous delivery had higher scores on openness to experience, agreeableness, and extroversion than women who had a normal and CS delivery (Table 2).

Investigation of the relationship between personality traits and the choice of delivery type based on educational level showed a significant difference between extraversion and openness to experience scores in terms of the level of education; nevertheless, there was no significant difference in other dimensions. Extraversion personality scores were higher in pregnant women with a high school diploma and openness to experience in pregnant women with an associate degree and above than in women with other educational levels (Table 2).

\section{Discussion}

The aim of the present study was to investigate the relationship between personality traits and type of delivery (C-section or normal) in pregnant women. CS and its complications have been increasing in most countries of the world, especially Iran, over the last few decades. Many domestic and international studies have focused on the factors affecting CS rates (2,13-15). Reviewing most of the previous domestic studies showed that most of them have been conducted in different cities and provinces across the country and have been limited to one or more hospitals. The existing studies have taken a medical approach and have less focused on comprehensive social, demographic, and attitudinal issues affecting the choice of delivery type. CS risk factors are mostly due to nonobstetric factors, among which fear of labor pain is the most important $(1,2,12)$. Since labor has a painful nature, mothers' reaction to labor pain varies depending on factors such as culture, fear and worry, previous delivery experience, readiness for delivery, and spouse 
support (6). Fear of labor pain has been identified as an important contributor to the decrease in vaginal delivery and increased CS rate, and maternal personality traits can play a significant and influential role in the development of such fear (10). A study (2013) aimed to develop a multi-component instrument to evaluate decisions made by Taiwanese pregnant women regarding normal and CS deliveries based on the theory of planned behavior. The results suggested that decision making about delivery options may be moderated by interventions for pregnant women's attitudes toward family and culture-based prenatal programs (12).

The participants of the present study were divided into five personality groups as follows: extroversion, openness to experience, agreeableness, conscientiousness, and neuroticism.

The results showed that the rate of vaginal delivery was higher among extraversion and conscientiousness groups than that of CC and there is a significant relationship between personality traits and the choice of delivery type.

Interestingly, a specific tendency to infant's gender was seen only among women with neuroticism and openness to experience personality traits so the tendency to have a female and male infant was higher in the former and latter, respectively; however, this relationship was not significant in other groups.

The present study showed a significant difference between neuroticism $(P$ value $=0.003)$ and openness to experience $(P$ value $=0.39)$ in terms of the choice of the infant's gender; however, the difference was not statistically significant in other dimensions. Pregnant women with higher mean neuroticism scores tended to have a female infant and women who had higher scores on openness to experience had a tendency to have a male infant. Similar results have been obtained in other studies $(6,16)$.

There was a statistically significant difference between openness to experience, agreeableness and extraversion personality traits in terms of the choice of delivery type based on the patient's previous history of delivery, but the difference was not statistically different in other dimensions. Pregnant women who did not have previous history of delivery had higher scores on openness to experience, agreeableness, and extroversion dimensions than women who had normal and CS deliveries.

There was a significant difference between extraversion and openness to experience personality dimensions in terms of the choice of delivery type based on level education, while there was no significant difference in other dimensions. Extraversion personality scores were higher in pregnant women with a high school diploma and openness to experience scores in pregnant women with an associate degree and above compared to other educational levels. The present study showed a significant relationship between extraversion personality trait and age, while there was no significant relationship between age and other personality dimensions. Pregnant women who aged 15-25 years had higher extraversion scores than other age groups. The results of a study on the relationship between the personality traits and the previous pregnancy planning showed no significant difference in the choice of having one's husband in the labor, the place of residence, and any of the personality dimensions that were not investigated in previous similar studies (6).

Consistent with the present study, a relatively similar study aimed at comparing personality traits (neuroticism, extraversion, openness to experience, agreeableness, and conscientiousness) and anxiety (explicit and implicit) of pregnant women based on the choice of delivery type (normal or CS) using NEO-FFI. It showed significant difference between personality traits of neuroticism, extraversion, openness to experience, and accountability in pregnant women with choice of delivery type (normal and CS) in terms of the level of explicit anxiety. The results of similar studies emphasized the importance of psychological issues in the choice of delivery type and revealed that it is necessary to pay attention to the explicit anxiety and personality traits of pregnant women to reduce the number of CSs $(17,18)$

The present study showed that the mean extraversion scores were higher in patients who tended to have a vaginal delivery than in those who opted for CS, indicating that women who were sociable, warm, talkative, fun-loving, and more emotional were more likely to have a normal delivery type.

The results of another study aiming at assessing the relationship between personality traits and choice of type of delivery (vaginal or CS delivery) in 2016 showed a significant relationship between the neuroticism dimension and the type of delivery, which was consistent with the results of the present study (19). It can be concluded that the personality traits of individuals can determine the type of delivery by creating specific moods. In this regard, we can provide a framework for pregnant women's behavior regarding the choice of delivery type and reduce the CS rate by identifying the factors affecting these traits.

One of the strengths of the present research was to understand the importance of the role of personality traits and anxiety in pregnant women based on their choice of delivery type. Therefore, considering the importance of the subject and the necessity of identifying the personality traits and factors affecting them, we can play a significant role in decreasing the CS rate by providing necessary longterm training and changing the attitude of individuals.

\section{Conclusion}

The results of the current study indicated that personality traits can determine the type of delivery by creating specific moods. Therefore, considering the complications and the high rate of CS in Iran, we can play a role in decreasing the CS rate by providing necessary long-term training and 
changing individuals' attitude. It is recommended that the effectiveness of the aforementioned cases should be investigated in future studies. In addition, the relationship between the choice of delivery type and other constructs such as emotional intelligence, spiritual intelligence, resilience, and mental toughness should be investigated. Considering the complications and rate of CS in Iran, it is desirable to reduce the CS rate by changing the attitudes of individuals with different personality traits based on systematic and targeted training.

\section{Conflict of Interest Disclosures}

The authors have no conflict of interests to declare.

\section{Acknowledgments}

The authors would like to thank the Research Department of Hormozgan University of Medical Sciences for supporting the research as well as Psychology Department of Hormozgan University of Medical Sciences and the manager of Shahid Mohammadi Hospital.

\section{Ethical Statement}

This study was approved by the Ethics Committee of Hormozgan University of Medical Sciences (IR.HUMS.REC.1397.142).

\section{Authors' Contribution}

AM, MNM, HM, and Sayed HS collected the data. AM and ZMA developed the study design and edited the article. AM and ZMA and $\mathrm{HM}$ analyzed the data, interpreted the results, and prepared the manuscript. All authors read and approved the final version of the manuscript.

\section{Funding/Support}

This study was financially supported by the Research Department of Hormozgan University of Medical Sciences.

\section{Informed Consent}

The purpose of the study was explained to the participants before data collection and the informed consent was obtained verbally from them.

\section{References}

1. Fabri RH, de Souza Lima e Silva H, de Lima RV, Murta EFC. [Comparative study of cesarean section indications between a public university hospital and a private hospital]. Rev Bras Saúde Mater Infant. 2002;2(1):29-35. doi : 10.1590/S151938292002000100005

2. Harris LH. Counselling women about choice. Best Pract Res Clin Obstet Gynaecol. 2001;15(1):93-107. doi: 10.1053/ beog.2000.0151.

3. Goodwin A, Astbury J, McMeeken J. Body image and psychological well-being in pregnancy. A comparison of exercisers and non-exercisers. Aust N Z J Obstet Gynaecol. 2000;40(4):442-7. doi: 10.1111/j.1479-828x.2000. tb01178.x

4. Maroufizadeh S, Bagheri-Lankarani N, Almasi-Hashiani A, Amini P, Esmaeilzadeh A, Navid B, et al. Prevalence of cesarean section and its related factors among primiparas in Tehran province, Iran, in 2015. Journal of Isfahan Medical School. 2017;35(423):303-9. [Persian].

5. Amidi M, Ghofranipoor F, Hosseini R. Body image dissatisfaction and body mass index in adolescent girls. Journal of Research in Behavioral Sciences. 2006;1(4):59-65 .

6. Hejazi M. Comparison of personality characteristics and anxiety in pregnant women based on choosing the type of delivery. The Scientific Journal of Rehabilitation Medicine. 2018;7(1):51-8. doi: 10.22037/jrm.2018.110756.1508. [Persian].

7. Hofstede G. Culture's Consequences. Beverly Hills, CA: Sage; 1980.

8. Goldberg LR. The structure of phenotypic personality traits. Am Psychol. 1993;48(1):26-34. doi: 10.1037//0003066x.48.1.26.

9. Chervenak F."Let science and ethics guide primary elective c-section decisions". OB/GYN News 2004. http://findarticles. com/p/articles/mi_m0CYD/ is_13_39/ai_n6114105/ ( accessed on May 2009

10. Chervenak FA, McCullough LB, et al. "Ethical issues in offering, recommending, and performing cesarean delivery". http://HYPERLINK "http://www.uptodate.com/ patients/content/topic.do?topicKey= q1 quiOWZNfzauWD" www.uptodate.com/patients/content/topic. do?topicKey= q1 qviOWZNfzauWD. (accessed on Dec 2008)

11. McCrae RR, Costa PT Jr, Busch CM. Evaluating comprehensiveness in personality systems: the California Q-Set and the five-factor model. J Pers. 1986;54(2):430-46. doi: 10.1111/j.1467-6494.1986.tb00403.x.

12. Tai CY. Development of an Investigator-designed Questionnaire Concerning Childbirth Delivery Options based on the Theory of Planned Behavior. University of South Florida; 2013.

13. Gibbons L, Belizán JM, Lauer JA, Betrán AP, Merialdi M, Althabe F. The Global Numbers and Costs of Additionally Needed and Unnecessary Caesarean Sections Performed per Year: Overuse as a Barrier to Universal Coverage. World Health Report; 2010.

14. Gunnigham F, Mocdonald P, Leveno K, et al. (1993). Cesarean section and cesarean hysterectomy. Wiliams Obstetrics. Prentice Hail International, Inc.19: 591-594 .

15. Hamilton BE, Martin JA, Osterman MJ. Births: preliminary data for 2015. Natl Vital Stat Rep. 2016;65(3):1-15.

16. Franzoi SL, Herzog ME. Judging physical attractiveness: what body aspects do we use? Pers Soc Psychol Bull. 1987;13(1):1933. doi: $10.1177 / 0146167287131002$.

17. Ajzen I. The theory of planned behavior. Organ Behav Hum Decis Process. 1991;50(2):179-211. doi: 10.1016/07495978(91)90020-T.

18. Ajzen I. Perceived behavioral control, self-efficacy, locus of control, and the theory of planned behavior. J Appl Soc Psychol. 2002;32(4):665-83. doi: 10.1111/j.1559-1816.2002. tb00236.x.

19. Akbarzadeh F, Talaei A, Tavassoli F, Jalaliani S, Talebi M, Modarres Gharavi M, et al. Psychological characteristics of women with vaginal delivery, elective and emergency cesarean section. Medical Journal of Mashhad University of Medical Sciences. 2016;58(10):611-9. doi: 10.22038/ mjms.2016.6789. [Persian]. 